\title{
THE CATALAN EQUATION OVER FUNCTION FIELDS
}

\author{
BY
}

JOSEPH H. SILVERMAN

\begin{abstract}
Let $K$ be the function field of a projective variety. Fix $a, b, c \in K^{*}$. We show that if $\max \{m, n\}$ is sufficiently large, then the Catalan equation $a x^{m}+b y^{n}=c$ has no nonconstant solutions $x, y \in K$.
\end{abstract}

The Cassels-Catalan conjecture states that for fixed nonzero integers $a, b, c$, the equation $a x^{m}+b y^{n}=c$ has only finitely many solutions in integers $x, y, m, n$ satisfying $m \geqslant 3$ and $n,|x|,|y| \geqslant 2$. At present, the only known case of this conjecture is $a=-b=c=1$, due to Tijdeman [6]. In this paper we prove a strengthened version of the Cassels-Catalan conjecture in the case that the number field $\mathbf{Q}$ is replaced by an arbitrary function field. The proof uses only elementary algebraic geometry, the principal tool being the Riemann-Hurwitz formula.

THEOREM. Let $k$ be a field of characteristic $p$ ( possibly with $p=0$ ), and let $K / k$ be the function field of a nonsingular projective variety. Fix $a, b, c \in K^{*}$.

Then there are only finitely many pairs of integers $m, n \geqslant 2$ ( prime to $p$ if $p \neq 0$ ) for which the Cassels-Catalan equation $a x^{m}+b y^{n}=c$ has even a single nonconstant solution $x, y \in K, x, y \notin k$.

Further, for any particular pair $m, n$ as above, there will be only finitely many solutions $x, y \in K$ unless either:

(i) $a / c$ is an mth power and $b / c$ is an nth power in $K$, in which case there may be infinitely many solutions $(x, y)=\left(\alpha(a / c)^{1 / m}, \beta(b / c)^{1 / n}\right)$ with $\alpha, \beta \in k$ satisfying $\alpha^{m}+\beta^{n}=1 ;$ or

(ii) $(m, n) \in\{(2,2),(2,3),(3,2),(2,4),(4,2),(3,3)\}$, in which case the CasselsCatalan equation defines a curve of genus 0 or 1 over $K$.

Proof. We first note that taking "generic" hyperplane sections of the variety whose function field is $K$, we are reduced by Bertini's theorem to the case that $K=k(C)$ is the function field of a nonsingular projective curve $C$. (See, e.g., [5] for the details of this standard reduction.) Second, we may replace $k$ by its algebraic closure, since at worst this will create extra solutions. Third, dividing the equation by $c$ and replacing $a$ and $b$ by $a / c$ and $b / c$, we may assume that our equation is

$$
a x^{m}+b y^{n}=1 \text {. }
$$

Let $D=\max \{\operatorname{deg}(a), \operatorname{deg}(b)\}$. (Note $a$ and $b$ are now functions on the curve $C$, so have degrees.) By symmetry, we may assume that $m \geqslant n$.

Received by the editors May 28, 1981 and, in revised form, July 24, 1981.

1980 Mathematics Subject Classification. Primary 10B15; Secondary 10J06, 14J25.

(C)1982 American Mathematical Society $0002-9947 / 81 / 0000-0631 / \$ 02.25$ 
Consider the desingularization of the covering of $C$ given by the equations $u^{m}=a, v^{n}=b$. Since this is a composition of cyclic coverings (note that $k$ contains both an $m$ th and an $n$th root of unity), it will consist of a union of isomorphic curves. Let $C^{\prime}$ be any one of these curves, and $f: C^{\prime} \rightarrow C$ the natural map. We note for future reference that the degree of $f$ divides $m n$; in particular, $\operatorname{deg}(f)$ is prime to $p$ if $p>0$.

Let $V / C$ be the projective surface given by the equation $a x^{m}+b y^{n} z^{m-n}=z^{m}$. Let $V^{\prime}=V \times{ }_{C} C^{\prime}$ be base extension of $V$ by $C^{\prime}$. If $V_{0}$ is the projective curve $X^{m}+Y^{n} Z^{m-n}=Z^{m}$ then we have a natural map

$$
\begin{aligned}
V^{\prime} & \rightarrow V_{0}, \\
{[x, y, z] } & \rightarrow[u x, v y, z] .
\end{aligned}
$$

Further let $V_{0}^{*}$ be the desingularization of $V_{0}$ and $h: V_{0}^{*} \rightarrow V_{0}$ the natural map.

Now suppose that we are given functions $x, y \in k(C)$ which satisfy equation (*). Then $P=[s, y, 1]$ gives a section $P: C \rightarrow V$. This extends to a section $C^{\prime} \rightarrow V^{\prime}$, and composed with the map $V^{\prime} \rightarrow V_{0}$ from above, gives a map of curves $C^{\prime} \rightarrow V_{0}$. But $C^{\prime}$ is nonsingular, so it factors through the normalization map $h$ to yield a map $\phi$ : $C^{\prime} \rightarrow V_{0}^{*}$. All of this is summarized in the following diagram.

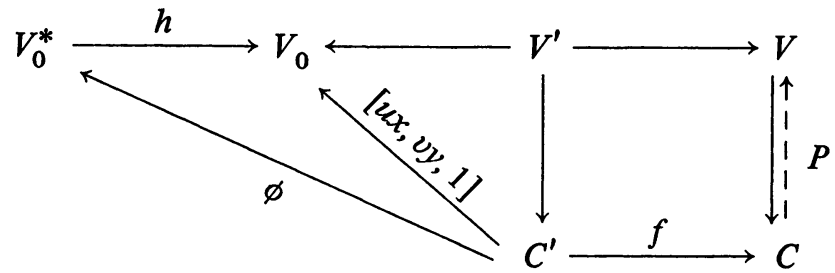

Assume for now that $\phi$ is surjective. (The other case is dealt with later.) We apply the Riemann-Hurwitz formula twice, once to $\phi$ and once to $f$. Note that since $\operatorname{deg}(f)$ is prime to $p=\operatorname{char}(k)$, there is no wild ramification in $f$ even if $p>0$. (See, e.g., [3, Chapter IV. 2] for basic facts about the Riemann-Hurwitz formula.)

$$
\begin{aligned}
& {\left[2 g\left(V_{0}^{*}\right)-2\right] } \operatorname{deg}(\phi) \leqslant 2 g\left(C^{\prime}\right)-2 \\
&=[2 g(C)-2] \operatorname{deg}(f)+\sum_{t \in C}\left[e_{t}(f)-1\right],
\end{aligned}
$$

where $e_{t}(f)=\operatorname{deg}(f)-\# f^{-1}(t)+1$. Now $e_{t}(f) \leqslant \operatorname{deg}(f)$, and the only points $t \in C$ at which $f$ can possibly ramify are zeros and poles of $a$ and $b$. Thus $e_{t}(f)=1$ except possibly at $4 D$ points of $C$, hence

$$
\sum_{t \in C}\left[e_{t}(f)-1\right] \leqslant 4 D \operatorname{deg}(f) \text {. }
$$

It is an elementary exercise to resolve the singularity of $V_{0}$ at $[0,1,0]$ (which is singular if and only if $m-n>1$ ) and compute the Euler characteristic of $V_{0}^{*}$. One finds

$$
2 g\left(V_{0}^{*}\right)-2=m n-m-n-(m, n),
$$

where $(m, n)$ is the greatest common divisor of $m$ and $n$. 
Next we express $\operatorname{deg}(\phi)$ in terms of $x$ and $y$. Consider the following commutative diagram.

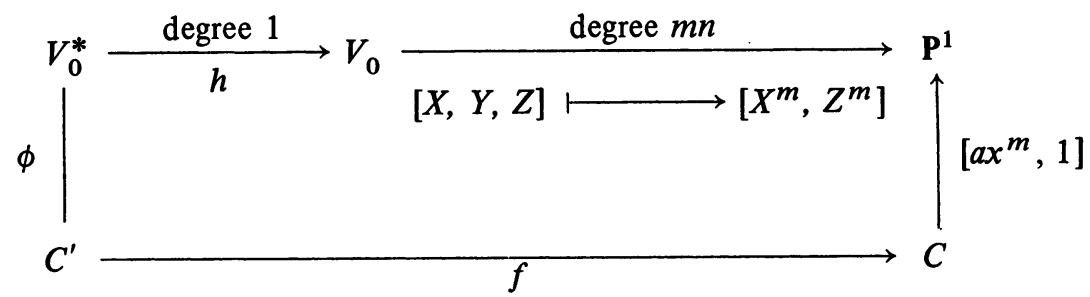

From this we read off

$$
\operatorname{deg}(\phi)=\frac{\operatorname{deg}\left(a x^{m}\right) \operatorname{deg}(f)}{m n} \geqslant \frac{\left(\operatorname{deg}\left(x^{m}\right)-D\right) \operatorname{deg}(f)}{m n} .
$$

Using a similar diagram we obtain

$$
\operatorname{deg}(\phi)=\frac{\operatorname{deg}\left(b y^{n}\right) \operatorname{deg}(f)}{m n} \geqslant \frac{\left(\operatorname{deg}\left(y^{n}\right)-D\right) \operatorname{deg}(f)}{m n} .
$$

Now putting all of these computations into the inequality $(* *)$ and dividing by $\operatorname{deg}(f)$, we obtain

$$
\begin{aligned}
\left(5-m^{-1}-n^{-1}-\right. & {\left.[m, n]^{-1}\right) D+2 g(C)-2 } \\
\geqslant & \left(1-m^{-1}-n^{-1}-[m, n]^{-1}\right) \max \left\{\operatorname{deg}\left(x^{m}\right), \operatorname{deg}\left(y^{n}\right)\right\},
\end{aligned}
$$

where $[m, n]$ is the least common multiple of $m$ and $n$. In particular we obtain the fundamental inequality

$$
\text { (+) } 5 D+2 g(C)-2 \geqslant\left(1-m^{-1}-n^{-1}-[m, n]^{-1}\right) \max \left\{\operatorname{deg}\left(x^{m}\right), \operatorname{deg}\left(y^{n}\right)\right\} \text {. }
$$

We now check the case that $\phi$ is a constant map. This will mean that $u x$ and $v y$ are constant functions on $C^{\prime}$, so raising to the $m$ th (respectively $n$ th) power we find that $a x^{m}$ and $b y^{n}$ are in the constant field $k$. Hence, since $a b \neq 0, \operatorname{deg}\left(x^{m}\right) \leqslant \operatorname{deg}(a) \leqslant D$ and $\operatorname{deg}\left(y^{n}\right) \leqslant \operatorname{deg}(b) \leqslant D$. These are stronger than the inequality (+) except in the one trivial case $D=g(C)=0$, in which case they imply that $\operatorname{deg}(x)=\operatorname{deg}(y)=0$, while $(+)$ would yield the impossibility $-2 \geqslant 0$. In any case, $(+)$ holds whenever $x$ and $y$ are not constant functions.

It is easy to check that if $m, n \geqslant 2$ are integers other than the six pairs listed in (ii) of the theorem, then

$$
1-1 / m-1 / n-1 /[m, n] \geqslant 1 / 6
$$

(attaining this minumum for $(m, n)=(6,2))$. Now since our solution $x, y \in k(C)$ to (*) was assumed to be nonconstant, we have $\operatorname{deg}(x), \operatorname{deg}(y) \geqslant 1$, so the inequality $(+)$ yields

$$
5 D+2 g(C)-2 \geqslant \frac{1}{6} \max \{m, n\} \text {. }
$$


This bounds the possibilities for $m$ and $n$. Then, for any particular $m$ and $n$,

$$
5 D+2 g(C)-2 \geqslant \frac{1}{6} \max \{m \operatorname{deg}(x), n \operatorname{deg}(y)\}
$$

bounds $\operatorname{deg}(x)$ and $\operatorname{deg}(y)$. But a curve of genus at least 2 over a function field $K$ can have only finitely many points of bounded degree unless the curve is birational, over $K$, to a curve defined over the field of constants $k$. Further, all but finitely many of thoses points will come from $k$-valued points on the new curve. (See [5, Corollary on p. 42]. Note that the result is true also for $\operatorname{char}(k)=p>0$, since we have taken $k$ to be algebraically closed. In general, one must take a purely inseparable extension of $k$.) One easily checks that the only way for the CasselsCatalan equation (*) to reduce to an equation over $k$ is for $a$ to be a $m$ th power and $b$ to be an $n$th power, which is the case covered by (i) of the theorem.

The inequality $(+)$ derived in the course of proving the above theorem is of independent interest, since it gives effective bounds for $m, n, \operatorname{deg}(x), \operatorname{deg}(y)$ in the case that $K$ is the function field of a curve. We therefore state it separately.

THEOREM. Let $k$ be a field and $C / k$ a nonsingular projective curve with function field $k(C)$. Fix two functions $a, b \in k(C)^{*}$. Suppose $m, n \geqslant 2$ are integers [prime to $\operatorname{char}(k)$ if $\operatorname{char}(k) \neq 0$ ] and $x, y \in k(C)$ are functions satisfying $a x^{m}+b y^{n}=1$. Then

$$
\begin{aligned}
5 \max \{\operatorname{deg}(a), \operatorname{deg}(b)\} & +2 g(C)-2 \\
\geqslant & \left(1-m^{-1}-n^{-1}-[m, n]^{-1}\right) \max \left\{\operatorname{deg}\left(x^{m}\right), \operatorname{deg}\left(y^{n}\right)\right\} .
\end{aligned}
$$

(except in the trivial case $g=\operatorname{deg}(x)=\operatorname{deg}(y)=\operatorname{deg}(a)=\operatorname{deg}(b)=0)$.

It is likely that the coefficient 5 in the left-hand side of this inequality can be improved, and one might ask what the best possible result is. In the special case that $g=0$ (so $k(C)=k(t)$ is a rational function field), if one restricts $x$ and $y$ to be polynomials, then Davenport has used very different arguments to obtain a similar inequality [1]. If $g=0, a=b=1$, and $m=n$ (i.e. the Fermat equation over $k(t)$ ), one can show the nonexistence of nonconstant solutions in $k(t)$ by an easy descent argument [2]. Both of these proofs, however, use the unique factorization of $k[t]$, so do not readily generalize to other functions fields.

Returning to the case of number fields, one is tempted to conjecture that an inequality of this sort should hold (with $2 g(C)-2$ replaced by some function involving the degree of the number field over $\mathbf{Q}$ ). From this the strengthened version of the Cassels-Catalan conjecture would follow formally as in the above proof. (Notice a corollary would be Fermat's last theorem for sufficiently large exponents!) A similar inequality, but only for integral rather than rational solutions, has been proposed by Lang and Waldschmidt [4, p. 213]. They also show how it would follow from a certain Baker-style diophantine inequality, but this unfortunately seems well beyond current techniques.

ACKNOWLEDGEMENT. I would like to thank Barry Mazur for his encouragement and helpful suggestions. 


\section{REFERENCES}

1. H. Davenport, $O n f^{3}(t)-g^{2}(t)$, Norske Vid. Selsk. Forh. (Trondheim) 38 (1965), 86-87.

2. N. Greenleaf, On Fermat's equation in $C(t)$, Amer. Math. Monthly 76 (1969), 808-809.

3. R. Hartshorne, Algebraic geometry, Graduate Texts in Math., vol. 52, Springer-Verlag, Berlin and New York, 1977.

4. S. Lang, Elliptic curves: Diophantine analysis, Grundlehren der Math. Wissenschaften, vol. 231, Springer-Verlag, Berlin and New York, 1978.

5. Integral points on curves, Inst. Hautes Études Sci. Publ. Math. 6 (1960), 27-43.

6. R. Tijdeman, On the equation of Catalan, Acta Arith. 29 (1976), 197-209.

Department of Mathematics, Harvard University, Cambridge, Massachusetts 02138 